\title{
Viscoelastic Flows in Simple Liquids Generated by Vibrating Nanostructures
}

\author{
Matthew Pelton, ${ }^{1, *}$ Debadi Chakraborty, ${ }^{2}$ Edward Malachosky, ${ }^{3}$ Philippe Guyot-Sionnest, ${ }^{3}$ and John E. Sader ${ }^{2,4, \dagger}$ \\ ${ }^{1}$ Center for Nanoscale Materials, Argonne National Laboratory, Argonne, Illinois 60439, USA \\ ${ }^{2}$ Department of Mathematics and Statistics, The University of Melbourne, Victoria 3010, Australia \\ ${ }^{3}$ James Franck Institute, University of Chicago, Chicago, Illinois 60637, USA \\ ${ }^{4}$ Kavli Nanoscience Institute and Department of Physics, California Institute of Technology, Pasadena, California 91125, USA
}

(Received 2 July 2013; published 13 December 2013)

\begin{abstract}
Newtonian fluid mechanics, in which the shear stress is proportional to the strain rate, is synonymous with the flow of simple liquids such as water. We report the measurement and theoretical verification of non-Newtonian, viscoelastic flow phenomena produced by the high-frequency $(20 \mathrm{GHz})$ vibration of gold nanoparticles immersed in water-glycerol mixtures. The observed viscoelasticity is not due to molecular confinement, but is a bulk continuum effect arising from the short time scale of vibration. This represents the first direct mechanical measurement of the intrinsic viscoelastic properties of simple bulk liquids, and opens a new paradigm for understanding extremely high frequency fluid mechanics, nanoscale sensing technologies, and biophysical processes.
\end{abstract}

DOI: 10.1103/PhysRevLett.111.244502

PACS numbers: 47.50. $-\mathrm{d}, 47.61 .-\mathrm{k}, 62.25 . \mathrm{Fg}, 87.15 . \mathrm{ht}$

A fluid is said to be Newtonian if it exhibits a simple linear relationship between shear stress and strain rate. This description, which underlies conventional fluid dynamics, provides an excellent approximation to the behavior of real fluids, provided the time scale for measurement is long compared to the time required for stresses to propagate in the fluid. In simple liquids, including water and glycerol, these "relaxation times" are on the order of 1 ps-1 ns [1-3]. These time scales are short compared to the time scales associated with the motion of macroscopic objects in the fluids, which allows interactions between solid structures and simple fluids to be described by classical Navier-Stokes treatments [4,5]. These treatments hold even for micrometer-scale objects, such as the cantilevers found in atomic force microscopes and microelectromechanical devices, because they have characteristic vibrational frequencies in the $\mathrm{kHz}$ to $\mathrm{MHz}$ range [6,7]. Non-Newtonian fluid mechanics is therefore conventionally associated only with complex fluids that have long relaxation times, such as polymer solutions and melts, dense colloidal suspensions such as corn starch in water, and fluids near their phase transitions [8,9]. Scaling objects down to nanometer size scales increases their characteristic vibrational frequencies up to the $\mathrm{GHz}$ or $\mathrm{THz}$ range [10]. Fluid-structure interactions on these length scales thus have the potential to show significant deviations from Newtonian behavior, even for simple liquids.

Departures from Newtonian behavior have been reported for simple liquids under extreme confinement, due to structural reorganization and surface effects on the molecular scale [11-13]. For bulk fluids, by contrast, direct mechanical observation of non-Newtonian behavior has been limited to solid structures interacting with dilute gases [14]. In this case, the effects can be predicted rigorously by the Boltzmann equation [15]. For simple bulk liquids, however, rigorous theoretical description of, and experimental access to, the non-Newtonian regime remain outstanding problems in the physical sciences.

We access this regime directly for the first time by exciting and probing vibrations in metal nanoparticles with ultrafast laser pulses [16-19]. In these experiments, absorption of a pump laser pulse leads to rapid heating and expansion of the nanoparticle, resulting in nearly impulsive excitation of coherent acoustic oscillations. These oscillations modulate the transmission of a second probe pulse due to changes in the resonance frequency of plasmons in the nanoparticles. Using highly monodisperse samples of bipyramidal gold nanoparticles [20,21] ensures narrow plasmon resonances, resulting in strong transient optical signals. It also minimizes the effects of inhomogeneous dephasing of the mechanical vibrations, so that energy damping times can be determined from the time-dependent measurements. The low volume fraction $\left(<10^{-6}\right)$ of the samples minimizes correlations between the particles. Previous measurements showed good agreement between the measured rate at which the nanoparticle vibrations are damped by surrounding low-viscosity solvents and models that treat the solvents as incompressible Newtonian fluids [22-24]. The models, however, could not account for the observed damping rates in high-viscosity liquids [24], and behavior in these liquids has previously had no explanation.

Here, we explore this high-viscosity regime by performing new measurements on bipyramidal gold nanoparticles in water-glycerol mixtures. Methods for nanoparticle synthesis, transient-absorption measurements, and data analysis follow our previous work [20,22,23] (see the Supplemental Material [25]). Representative nanoparticle images and transient-absorption data are shown in Figs. 1(a) and 1(b), and the measured results are 

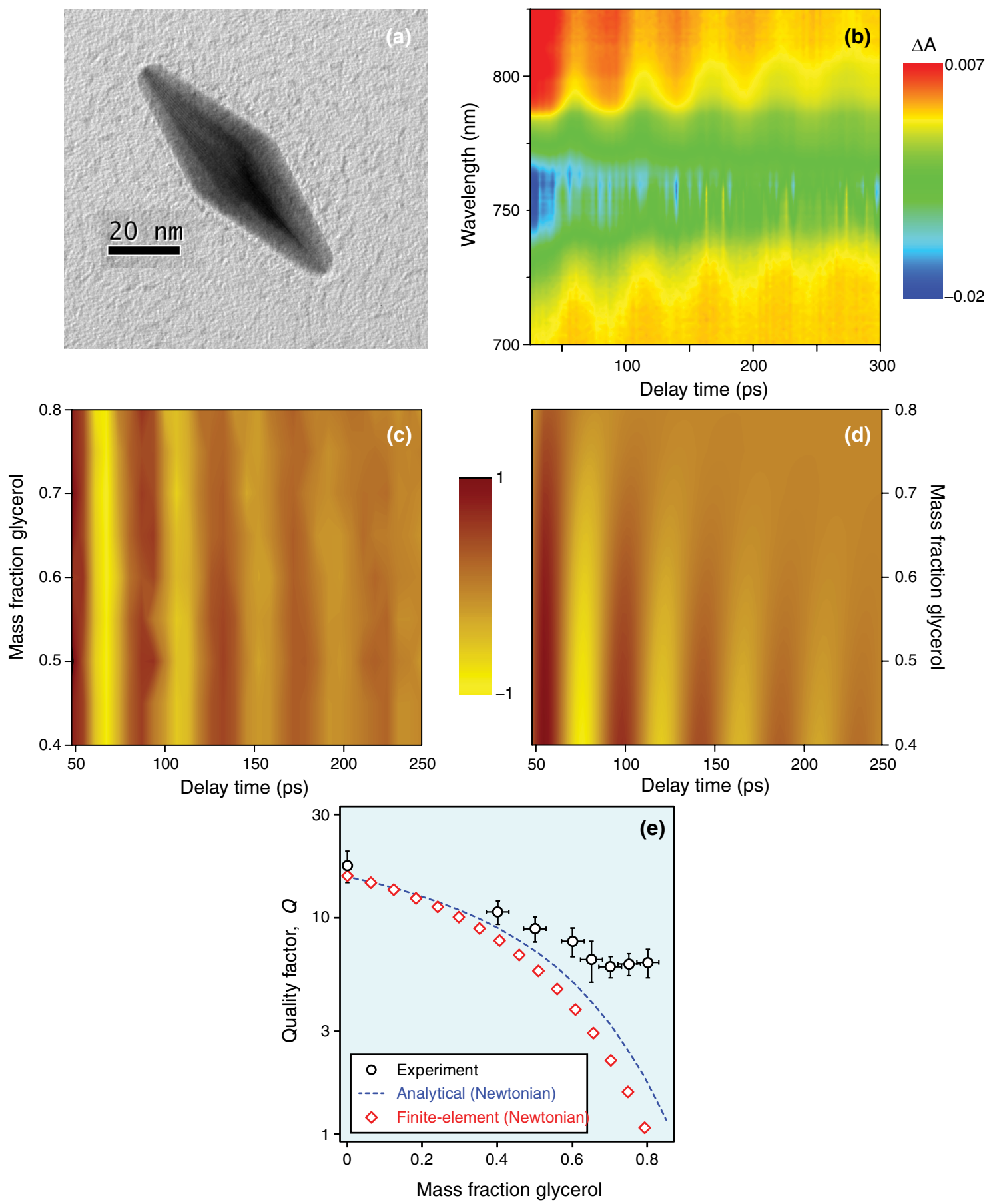

FIG. 1 (color online). Acoustic oscillations of bipyramidal gold nanoparticles. (a) Transmission-electron-microscope image of a representative gold bipyramid. (b) Representative transient-absorption data from an ensemble of gold bipyramids. Change in extinction $\Delta A$ is plotted as a function of pump-probe delay and probe wavelength. Results are shown for a mixture of $60 \%$ glycerol and $40 \%$ water by mass and for a pump-pulse energy of $360 \mathrm{~nJ}$. (c) Measured oscillations of the plasmon resonance frequency for bipyramids in glycerol-water mixtures. Frequency shifts were determined by fitting to transient-absorption data, the exponentially decaying background due to heating of the nanoparticles was removed, and the remaining shifts were normalized to have a minimum value of -1 . Results are shown for a pump-pulse energy of $120 \mathrm{~nJ}$. (d) Calculated oscillations of the plasmon resonance frequency for bipyramids in glycerol-water mixtures, plotted as in (c). The calculations are performed using an analytical model that treats the glycerol-water mixtures as Newtonian fluids. The initial phases of the oscillations are chosen to match the experimental results. (e) Quality factor $Q$ of mechanical oscillations of bipyramids in glycerol-water mixtures, as a function of the mass fraction of glycerol in the mixtures. Circles are determined by fitting experimental data; the dashed line is the result of an analytical model, assuming a quality factor due to intrinsic damping $Q_{\text {int }}=35$; diamonds are the results of finite-element calculations, assuming $Q_{\text {int }}=50$, consistent with previous measurements [24]. Both models treat the glycerol-water mixtures as Newtonian fluids. 

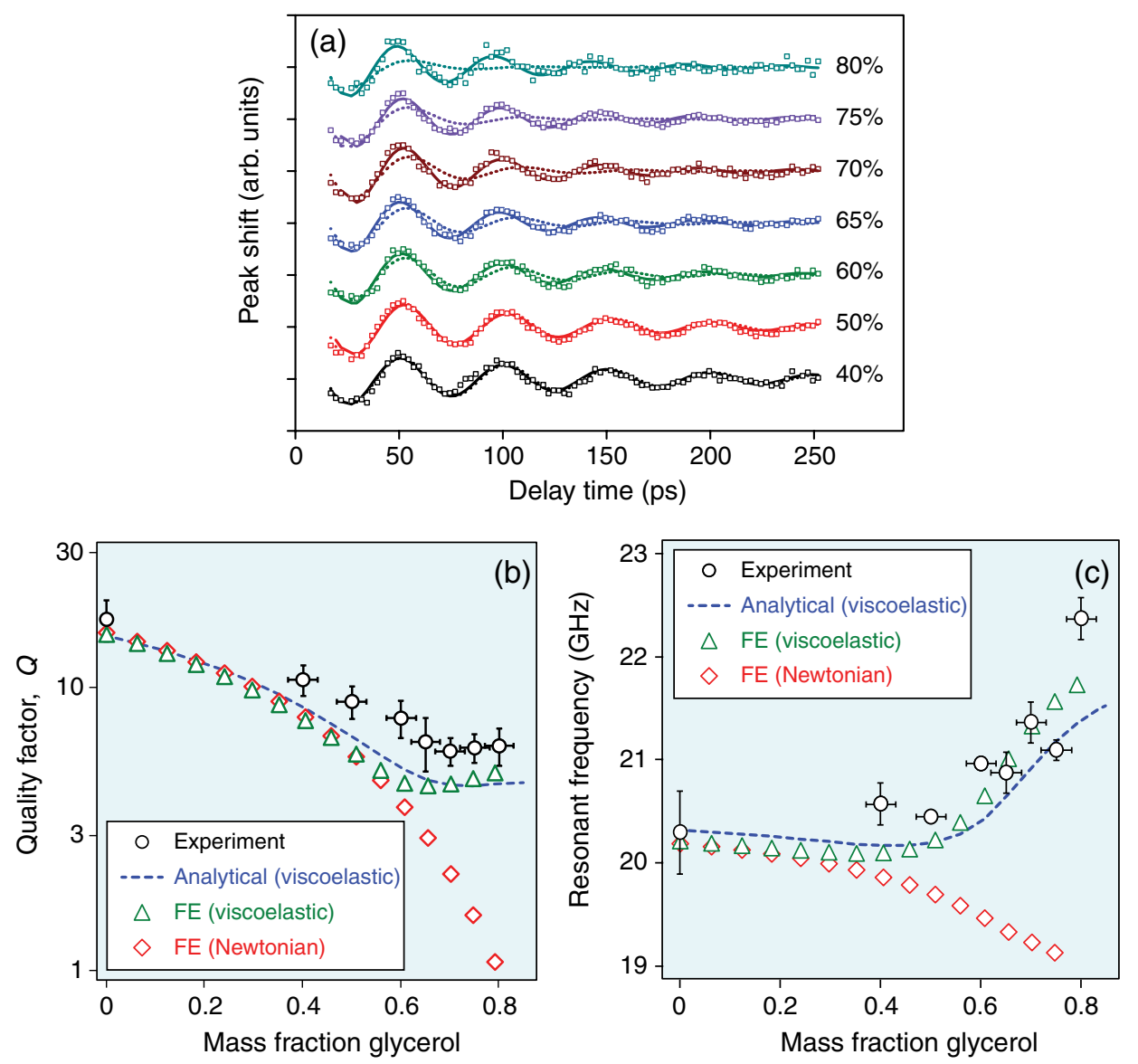

FIG. 2 (color online). Nanoparticle vibrations in Newtonian and viscoelastic liquids. (a) Oscillations in the plasmon resonance frequency for bipyramids in glycerol-water mixtures. Points are experimentally measured values for a pump-pulse energy of $120 \mathrm{~nJ}$ [see Fig. 1(c)]. Lines are the results of analytical calculations, with initial phases chosen to match the experimental results. Dashed lines are based on treating the glycerol-water mixtures as Newtonian fluids, and solid lines are based on treating them as viscoelastic fluids, described by a Maxwell model. Traces are offset vertically for clarity. (b) Quality factor $Q$ and (c) frequency of mechanical vibrations of bipyramids in glycerol-water mixtures. Circles are determined by fitting experimental data; the dashed line is the result of an analytical model that treats the glycerol-water mixtures as viscoelastic fluids, described by a Maxwell model; triangles are the result of finite-element (FE) calculations that treat the mixtures as viscoelastic fluids, and diamonds are the result of FE calculations that treat the mixtures as Newtonian fluids. Analytical calculations assume a quality factor due to intrinsic damping, $Q_{\text {int }}=35$, and FE calculations assume $Q_{\text {int }}=50$ [24].

summarized in Fig. 1(c). The data are first compared to an analytical model based on Newtonian fluid-structure theory [24,25]. As shown in Fig. 1(d), this model predicts strong damping as the viscosity of the mixtures increases. This is in striking disagreement with the observations, which show little change in the damping rates for glycerol fractions between $40 \%$ and $80 \%$.

The discrepancy between experiment and the analytical Newtonian model can be made more explicit by quantifying the damping rate in terms of the quality factor $Q=\omega_{\text {res }} T / 2$, where $\omega_{\text {res }}$ is the angular vibrational resonance frequency and $T$ is the time constant for damping of the oscillations. As shown in Fig. 1(e), the model reproduces experimental quality factors well for lower-viscosity mixtures, up to a glycerol mass fraction of approximately $60 \%$. At higher glycerol concentrations, however, the theoretical modeling completely fails to reproduce the experimental trend.

Figure 1(e) also shows the results of rigorous finiteelement simulations that assume Newtonian fluid mechanics [24,25]. The predictions of these calculations deviate only slightly from those of the analytical theory, due to pressure effects at the particle ends. Including the effects of fluid compressibility gives similar results, with the quality factor decreasing monotonically as the glycerol concentration rises (see the Supplemental Material [25]).

One might suspect that theory and experiment disagree because both the analytical model and the finite-element calculations use the conventional no-slip boundary condition at the liquid-solid interface. This condition has come under scrutiny in recent years, with a number of studies suggesting that it may be violated at nanometer length 
scales $[12,26]$. To assess the impact of such a violation, we adjust the calculations to allow for an arbitrary amount of slip. We find that the calculated quality factors still decrease monotonically as the solution viscosity is increased, so that this assumption cannot be responsible for the disagreement between theory and experiment (see the Supplemental Material [25]).

The remaining critical assumption involved in the theoretical models is that the fluid is Newtonian. The validity of this assumption can be quantified in terms of the Deborah number, $D e=\omega_{\text {res }} \lambda$, where $\lambda$ is the shear relaxation time of the fluid: elastic effects can be ignored only for De $\ll 1$ [8]. For the bipyramids used here, the longitudinal vibration period is approximately 50 ps [see Fig. 1(c)]. This means that $\mathrm{De} \approx 1$ for vibrating bipyramids in a waterglycerol mixture with a glycerol mass fraction of $60 \%$ [1]. For higher glycerol concentrations, strong viscoelastic effects and deviations from Newtonian behavior can be expected.

In the simplest model of a viscoelastic fluid subject to small strains, known as the linear Maxwell model, viscous and elastic effects combine in series [8],

$$
\mathbf{T}=-p \mathbf{I}+\mathbf{S}, \quad \mathbf{S}+\lambda \frac{\partial \mathbf{S}}{\partial t}=2 \mu \mathbf{D},
$$

where $\mathbf{T}$ is the stress tensor, $p$ is the pressure, $\mathbf{I}$ is the identity tensor, $\mathbf{S}$ is the deviatoric stress tensor as specified in the second equation, $\mathbf{D}$ is the rate-of-strain tensor, $t$ is time, and $\mu$ is the shear viscosity. Conceptually, the behavior of the Maxwell model can be visualized as that of a linear spring, with spring constant $k$, linked at its end to a viscous damper, with damping coefficient $b$; the system recovers from sudden deformation with a characteristic time $\lambda=b / k$. The Maxwell constitutive equation can readily be incorporated into the theoretical models (see the Supplemental Material [25]). As shown in Figs. 2(a) and 2(b), the resulting predictions reproduce the experimentally observed quality factors.

Strikingly, the viscoelastic model predicts a vibration frequency that increases with high glycerol content. This is in agreement with the experimental results, as shown in Fig. 2(c). By contrast, the Newtonian model always predicts a vibration frequency that decreases monotonically with increasing glycerol content. This qualitative difference provides clear evidence that the mechanical behavior of the nanoparticles is strongly affected by non-Newtonian fluid mechanics.

The nearly constant quality factor and increasing resonance frequency observed for increasing glycerol concentration can be understood as a consequence of the increasing importance of liquid elasticity. Quantitatively, $Q=2 \pi\left(E_{\text {stored }} / E_{\text {diss }}\right)$, where $E_{\text {stored }}$ is the maximum energy stored and $E_{\text {diss }}$ is the energy dissipated per cycle, with both quantities evaluated at resonance. At low glycerol concentrations, where De $\ll 1$, the elastic component of the liquid response exerts a negligible effect, and energy

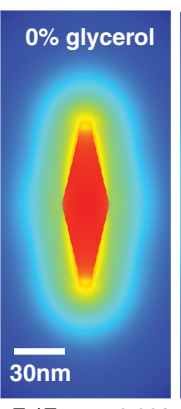

$E_{f} / E_{\text {TOTAL }}=0.003$

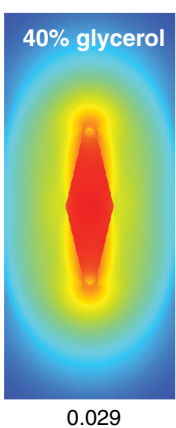

0.029

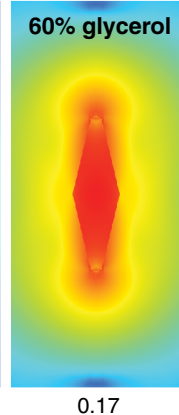

0.17

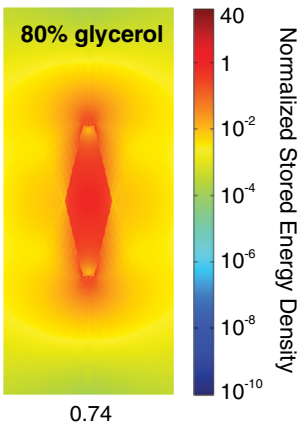

0.74
FIG. 3 (color online). Maximum energy density stored in a vibrating gold nanoparticle and in surrounding water-glycerol mixtures. The color scale indicates the stored energy density, normalized by the energy density in the center of the nanoparticle. Results are obtained from finite-element calculations that treat the particles as linear elastic solids and the liquids as viscoelastic Maxwell fluids. The fraction of the total energy stored in the fluid is indicated below each spatial plot.

is stored only in the solid nanoparticle. In this Newtonian limit, increasing the glycerol concentration thus only raises the viscosity and increases $E_{\text {diss }}$, resulting in lower $Q$. For higher glycerol concentrations, however, where De $\geq 1$, energy can also be stored elastically in the liquid. As shown in Fig. 3, increasing the glycerol concentration in this viscoelastic regime leads to more energy being stored in the liquid. This, in turn, increases the total $E_{\text {stored }}$, compensating for the increase in $E_{\mathrm{diss}}$ as the viscosity increases. The quality factor thus no longer decreases with increasing glycerol content, and can even increase, as observed in Fig. 2(b). The additional stored energy also increases the total stiffness of the fluid-structure interaction, and is thus responsible for the rise in vibrational frequency shown in Fig. 2(c).

The good quantitative agreement between theory and measurement validates the use of a simple Maxwell model to describe viscoelastic effects in liquid-nanoparticle interactions. It also provides strong support for the theoretical description of these nanoscale systems using continuum mechanics. In particular, there is no need to invoke molecular effects or to depart from no-slip boundary conditions, in clear contrast to previous reports of nonNewtonian behavior in liquids under extreme confinement [11-13]. Likewise, molecular ordering of the liquid and/or a change in conformation of the polystyrene sulphonic acid (PSS) layer surrounding the particle surface cannot account for our observation (see the Supplemental Material [25]). The small discrepancies between theory and experiment may be due to limitations of the simple Maxwell model: instead of a single relaxation time, the response of the glycerol-water mixtures may better be described by a spectrum of relaxation times [1]. Furthermore, any shortcomings in tabulated relaxation times [1] will clearly affect the comparison. In fact, the current nanoparticle experiments provide independent measurements of these relaxation times. Unlike the 
ultrasound or inelastic-scattering methods previously used [1-3], nanoparticle vibrations provide a direct, mechanical probe of non-Newtonian effects in simple liquids. For smaller objects with higher vibrational frequencies, viscoelastic effects are expected to become important even for lower-viscosity liquids with faster shear relaxation times. Measurements of vibrations in gold nanoparticles with diameters close to $1 \mathrm{~nm}$, for example, have shown vibrational periods of less than 0.5 ps $[27,28]$, which means that they should induce strong viscoelastic effects in pure water.

The experiments reported here represent the first time that the intrinsic viscoelasticity of simple bulk liquids is observed to affect the mechanical response of vibrating solids. While high-viscosity liquids can induce strong damping in large-scale solids, simple liquids exhibit a strong elastic response at the intrinsically high frequencies of nanometer-sized structures, reducing the efficacy of this dissipation mechanism. Such effects should be general to liquid-structure interactions on the nanoscale [29,30]. This will have important implications for the development of a new generation of high-speed and high-sensitivity molecular and biomolecular sensors based on the mechanical vibrations of nanoscale oscillators operating in liquid [31]. It also means that the wealth of knowledge developed for macro- and microscale non-Newtonian flows of complex fluids can now be brought to bear on the nanoscale fluid mechanics of simple liquids.

We thank D. Gosztola for valuable assistance with the transient-absorption measurements. This work was performed, in part, at the Center for Nanoscale Materials, a U.S. Department of Energy, Office of Science, Office of Basic Energy Sciences User Facility under Contract No. DE-AC02-06CH11357. E. M. was supported by NSF Grant No. CHE1111799. This research was supported by the Australian Research Council Grants Scheme and by Caltech's Kavli Nanoscience Institute.

* Present address: Department of Physics, University of Maryland, Baltimore County, Baltimore, MD 21250, USA.

To whom all correspondence should be addressed. jsader@unimelb.edu.au

[1] W. M. Slie, A. R. Donfor, and T. A. Litovitz, J. Chem. Phys. 44, 3712 (1966).

[2] G. Maisano, P. Migliardo, F. Aliotta, C. Vasi, F. Wanderlingh, and G. D'Arrigo, Phys. Rev. Lett. 52, 1025 (1984).

[3] F. Sette, G. Ruocco, M. Krisch, U. Bergmann, C. Masciovecchio, V. Mazzacurati, G. Signorelli, and R. Verbeni, Phys. Rev. Lett. 75, 850 (1995).
[4] H.J. Bungartz and M. Schäfer, Fluid-Structure Interaction: Modeling, Simulation, Optimization (Springer, Berlin, 2006).

[5] M.P. Païdoussis, Fluid-Structure Interactions: Slender Structures and Axial Flow, Vol. 1 (Elsevier, Holland, 2003).

[6] J.E. Sader, J. Appl. Phys. 84, 64 (1998).

[7] C. P. Green and J. E. Sader, J. Appl. Phys. 92, 6262 (2002).

[8] W. R. Schowalter, Mechanics of Non-Newtonian Fluids (Pergamon, Oxford, 1978).

[9] F. A. Morrison, Understanding Rheology (Oxford University Press, Oxford, 2001).

[10] A. N. Cleland, Foundations of Nanomechanics (Springer, Berlin, 2003).

[11] J. N. Israelachvili, P. M. McGuiggan, and A. M. Homola, Science 240, 189 (1988).

[12] S. Granick, Y.X. Zhu, and H. Lee, Nat. Mater. 2, 221 (2003).

[13] A. Labuda, K. Kobayashi, K. Suzuki, H. Yamada, and P. Grütter, Phys. Rev. Lett. 110, 066102 (2013).

[14] K. L. Ekinci, D. M. Karabacak, and V. Yakhot, Phys. Rev. Lett. 101, 264501 (2008).

[15] L.D. Landau and E.M. Lifshitz, Physical Kinetics (Butterworth-Heinemann, Oxford, 1981).

[16] C. Voisin, N. Del Fatti, D. Christofilos, and F. Vallée, J. Phys. Chem. B 105, 2264 (2001).

[17] G. V. Hartland, Annu. Rev. Phys. Chem. 57, 403 (2006).

[18] G. V. Hartland, Chem. Rev. 111, 3858 (2011).

[19] P. Ruijgrok, P. Zijlstra, A. L. Tchebotareva, and M. Orrit, Nano Lett. 12, 1063 (2012).

[20] M. Liu and P. Guyot-Sionnest, J. Phys. Chem. B 109, 22192 (2005).

[21] J. Burgin, M. Liu, and P. Guyot-Sionnest, J. Phys. Chem. C 112, 19279 (2008).

[22] M. Pelton, J.E. Sader, J. Burgin, M. Liu, P. GuyotSionnest, and D. Gosztola, Nat. Nanotechnol. 4, 492 (2009).

[23] M. Pelton, Y. Wang, D. Gosztola, and J. E. Sader, J. Phys. Chem. C 115, 23732 (2011).

[24] D. Chakraborty, E. van Leeuwen, M. Pelton, and J. E. Sader, J. Phys. Chem. C 117, 8536 (2013).

[25] See Supplemental Materials at http://link.aps.org/ supplemental/10.1103/PhysRevLett.111.244502 for materials and methods, derivation of theoretical models, finite-element calculations, and tests of assumptions.

[26] C. Neto, D. R. Evans, E. Bonaccurso, H.-J. Butt, and V. S. J. Craig, Rep. Prog. Phys. 68, 2859 (2005).

[27] V. Juvé, A. Crut, P. Maioli, M. Pellarin, M. Broyer, N. Del Fatti, and F. Vallée, Nano Lett. 10, 1853 (2010).

[28] O. Varnavski, G. Ramakrishna, J. Kim, F. Lee, and T. Goodson III, ACS Nano 4, 3406 (2010).

[29] T. M. Squires and S. R. Quake, Rev. Mod. Phys. 77, 977 (2005).

[30] J. T. C. Eijkel and A. van den Berg, Microfluid. Nanofluid. 1, 249 (2005).

[31] J.L. Arlett, E. B. Myers, and M. L. Roukes, Nat. Nanotechnol. 6, 203 (2011). 